\title{
Disordering and Melting of Aluminum Surfaces
}

Stoltze, Per; Nørskov, Jens Kehlet; Landman, U.

Published in:

Physical Review Letters

Link to article, DOI:

10.1103/PhysRevLett.61.440

Publication date:

1988

Document Version

Publisher's PDF, also known as Version of record

Link back to DTU Orbit

Citation (APA):

Stoltze, P., Nørskov, J. K., \& Landman, U. (1988). Disordering and Melting of Aluminum Surfaces. Physical Review Letters, 61(4), 440-443. https://doi.org/10.1103/PhysRevLett.61.440

\section{General rights}

Copyright and moral rights for the publications made accessible in the public portal are retained by the authors and/or other copyright owners and it is a condition of accessing publications that users recognise and abide by the legal requirements associated with these rights.

- Users may download and print one copy of any publication from the public portal for the purpose of private study or research.

- You may not further distribute the material or use it for any profit-making activity or commercial gain

- You may freely distribute the URL identifying the publication in the public portal

If you believe that this document breaches copyright please contact us providing details, and we will remove access to the work immediately and investigate your claim. 


\title{
Disordering and Melting of Aluminum Surfaces
}

\author{
P. Stoltze and J. K. Nørskov \\ Laboratory of Applied Physics II, Technical University of Denmark, DK-2800 Lyngby, Denmark, and \\ Haldor Topsøe Research Laboratories, DK-2800 Lyngby, Denmark
}

and

U. Landman

School of Physics, Georgia Institute of Technology, Atlanta, Georgia 30332

(Received 2 February 1988)

\begin{abstract}
We report on a molecular-dynamics simulation of an Al(110) surface using the effective-medium theory to describe the interatomic interactions. The surface region is found to start melting $\simeq 200 \mathrm{~K}$ below the bulk melting temperature with a gradual increase in the thickness of the disordered layer as the temperature approaches the bulk melting point. The more close-packed $\mathrm{Al}(111)$ surface shows a much weaker disordering below the melting temperature.
\end{abstract}

PACS numbers: $64.70 . \mathrm{Dv}, 68.35 . \mathrm{Md}$, 82.65.Dp

The understanding of the melting of a solid at the microscopic level is an outstanding problem in condensedmatter physics. The problem is presently receiving renewed interest because new experimental evidence has appeared, pointing to the importance of the surface in the melting process. ${ }^{1-3}$ It has been found that the (110) surfaces of $\mathrm{Pb}$ and $\mathrm{Al}$ show a beginning disordering of the outermost layer even at $150 \mathrm{~K}$ below the bulk melting temperature $T_{M}$, and a gradual thickening of the melted layer at temperatures just below $T_{M}$. This is the first microscopic evidence for the surface as the nucleation center for the melting process. The idea that this should be so is old, though, and macroscopic evidence has been known for years. ${ }^{4}$

Theoretically, the role of surfaces in the melting process has been discussed in terms of simple lattice instability arguments, ${ }^{5}$ and Landau theory. ${ }^{6}$ Surface premelting has also been found in computer simulations that use Lennard-Jones potentials to describe the interactions. $^{7,8}$ Lennard-Jones potentials give a reasonable description of inert-gas crystals, but are known to describe metals very poorly. This is particularly true for surfaces where the surface energies are not well described, and the relaxations of the interlayer spacings are generally of the wrong sign. Consequently, it is not clear that the phenomena observed for Lennard-Jones crystals have any bearing on metals.

Carnevalli, Ercolessi, and Tosatti ${ }^{9}$ have applied an empirical potential that includes many-body interactions to the melting of the Au(111) surface, and found that the reconstructed surface did not show any premelting, whereas the two outermost layers melt on the unreconstructed surface.

In the present Letter we present a molecular-dynamics simulation of the behavior of the $\mathrm{Al}(110)$ and (111) surfaces as they approach the melting point using an $a b$ initio effective-medium interaction potential. The effective-medium theory ${ }^{10}$ provides an approximate total energy expression which is ab initio in the sense that the functional form is derived with use of density-functional theory, and the parameters entering it can be calculated in the local-density approximation for exchange and correlation effects. It includes the many-body aspects of the interactions in a metallic system, and is still simple enough that it can be used in simulations.

The purpose of the Letter is threefold. We show that with a realistic metal potential that it is possible to give a (semi-) quantitative account of the experimental observations. We find a definite disordering of the outermost layers of $\mathrm{Al}(110)$ up to $200 \mathrm{~K}$ below the bulk melting point. The calculated temperature dependence of the LEED structure factor agrees very well with experiment. ${ }^{2}$ We also find that the close-packed (111) surface shows much weaker disordering than the (110) face. Secondly, we present new evidence that melting occurs as a layer-by-layer process. By calculating an order parameter for each layer as a function of temperature, we find that the temperature where a given layer disorders increases with depth. Finally, we make the observation that the tendency to disorder seems to be related to the energetic cost of making a vacancy.

The dynamics is studied in the adiabatic approximation. Normally the calculation of the adiabatic potential requires a self-consistent solution of the electronicstructure problem. Solutions are now available for special high-symmetry systems based on the local-density approximation for exchange and correlation effects, which give a very accurate description of the groundstate properties. ${ }^{11}$ These methods are, however, far too time consuming to allow dynamical simulations of reasonable-size systems.

The basic feature of the effective-medium theory ${ }^{10}$ is that the effect of the surroundings on a given atom in the system studied is parametrized. The time-consuming electron-structure problem can then be solved for each type of atom as a function of the external parameter. 


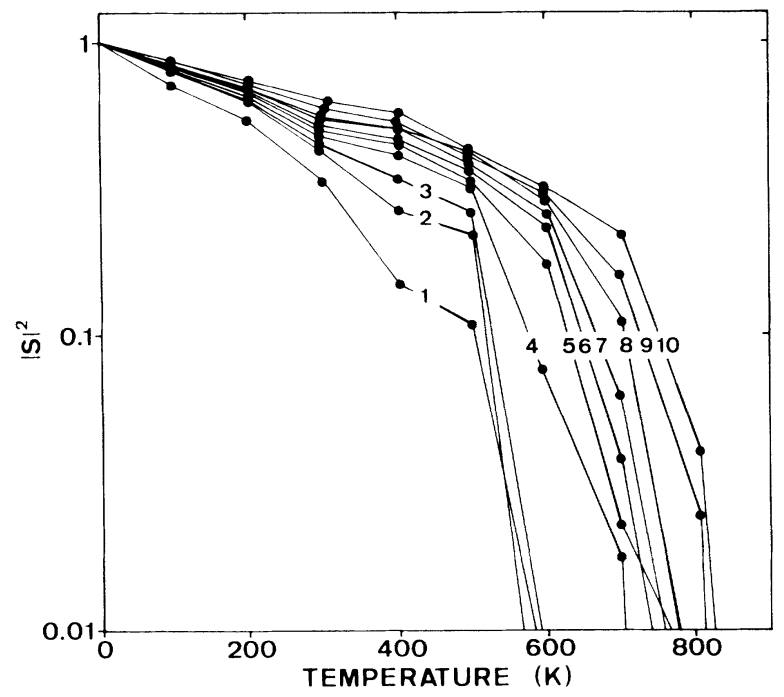

FIG. 1. The absolute square of the structure factor of Eq. (1) for layers 1 to 10 as a function of temperature for an $\mathrm{Al}(110)$ surface. The sudden decrease between 500 and $800 \mathrm{~K}$ is an indication of a loss of order which we associate with a melting transition.

The simplest parameter describing the surroundings in a metallic system is the average electron density provided by the neighbors of the atom in question. The electronic-structure problem is then that of an atom embedded in a homogeneous electron gas. This leads to the concept of a universal density-dependent energy function describing, to a first approximation, the energetics of this atom in any host. The density-dependent term gives rise to many-body interactions except if the energy function is linear in the density (which is written as a superposition of densities centered at the nuclear positions). Only for the rare gases is this approximately the case.

For a full derivation of the effective-medium expression for the total energy of a system of interacting atoms we refer to Ref. 10. We use Eq. (7.21) with the parameters for $\mathrm{Al}$ quoted in section VII.C. They are deduced from a calculation for $\mathrm{Al}$ in a homogeneous electron gas within the local-density approximation. ${ }^{12}$ The theory gives a good description of the bulk cohesive energy, lattice parameter, bulk modulus, and phonon spectrum and of the surface energies and surface lattice relaxations. ${ }^{10}$ Furthermore, a Monte Carlo simulation of the finitetemperature properties of the bulk metal gives a good description of the thermal expansion coefficient. ${ }^{13}$ There are thus good reasons to believe that it will provide a reasonable description of the surface properties at elevated temperatures. The approximate nature of the potential does, however, mean that we generally pay more attention to qualitative results and trends than to the absolute numbers that emerge.

The molecular-dynamics simulations have been performed with use of the velocity Verlet algorithm and Andersen thermalization. ${ }^{14}$ They were made on a semiinfinite $\mathrm{Al}$ crystal, where the deeper-lying layers are kept rigid with a lattice constant determined in an independent bulk calculation at the temperature in question. The system has been equilibrated at each temperature for 4 ps (around 40 lattice vibrations). After this time all system properties are completely stable, except for thermal fluctuations. An increase of the thermalization time by a factor of 2 or 4 does not change the results.
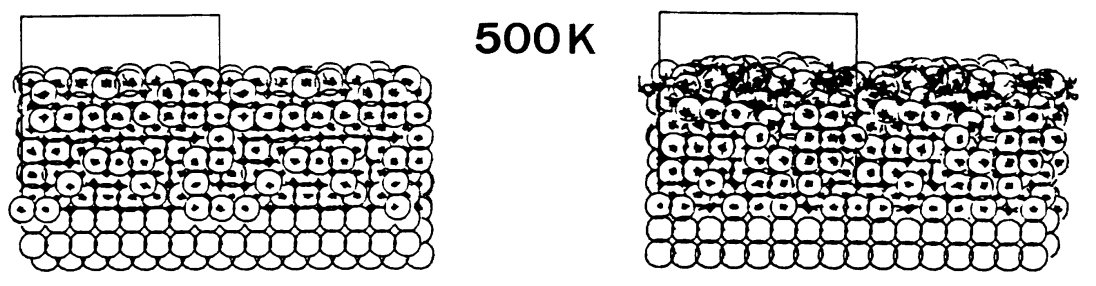

\section{$600 \mathrm{~K}$}
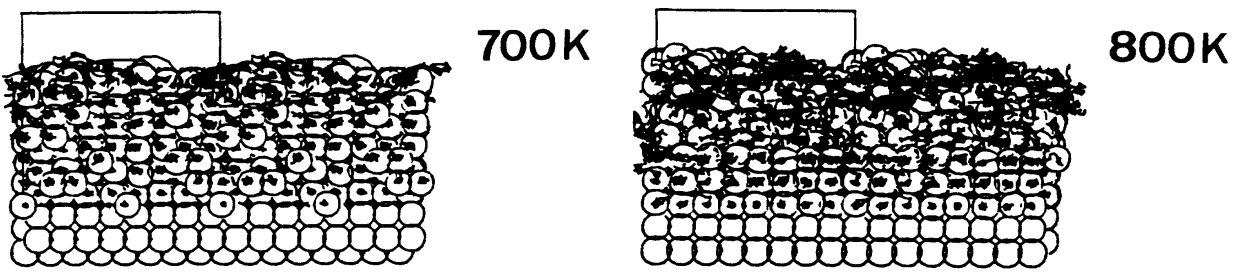

FIG. 2. The calculated atom trajectories for an $\mathrm{Al}(110)$ surface at various temperatures. The atoms are shown as circles in the initial (after thermalization) configuration and the trajectories are indicated by the nuclear positions. 


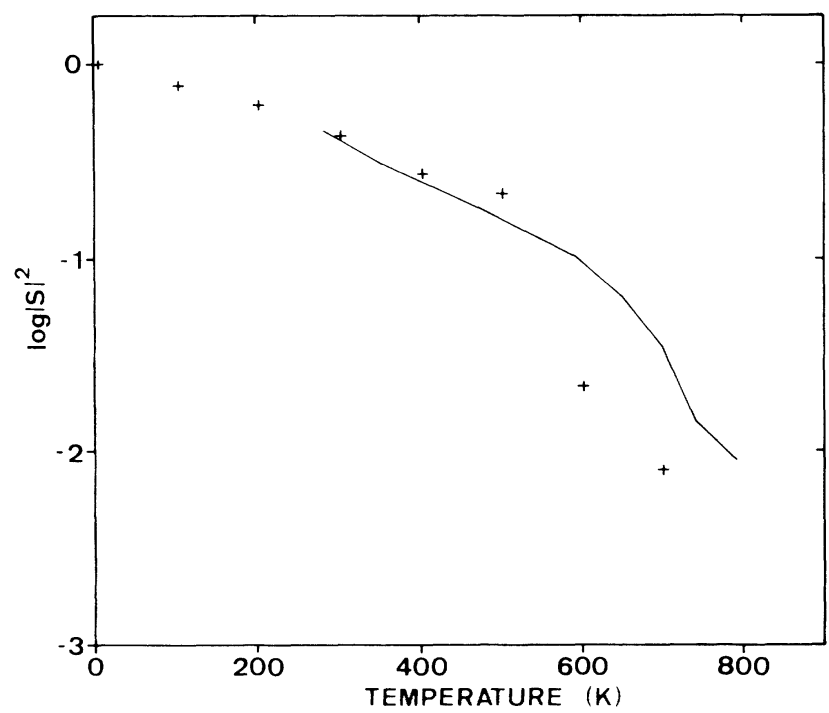

FIG. 3. The square of the LEED structure factor Eq. (2) (crosses) compared with the experimental values of Ref. 2 (full line).

Thermal averages are then made over an additional 4 ps. Again the results do not change if the time is doubled. Only density contributions from nearest neighbors are included. This is done by a gradual cutoff of the density tails between the nearest- and the next-nearest-neighbor distances. Because the densities fall off rapidly over one screening length this is a very good approximation, and it is found to have only a minor effect on the lowtemperature properties and the thermal expansion. The results presented here for $\mathrm{Al}(110)$ are obtained for a system with 12 dynamical and 4 static layers. Parallel to the surface periodic boundary conditions have been applied with 32 atoms in each layer. The qualitative results are found not to change if the number of dynamical layers or the number of atoms in each layer is halved.

The results are summarized in Figs. 1-3. In Fig. 1 we show the temperature dependence of the absolute square of the layer-by-layer structure factor

$$
\begin{aligned}
S(k, l, T)=\left\langle\sum_{i}\right. & \exp \left(i k r_{i}\right) \\
& \left.\times\left[\theta\left(z_{i}-z_{l-1}\right)-\theta\left(z_{i}-z_{l}\right)\right]\right\rangle .
\end{aligned}
$$

Here $l$ denotes the layer between $z_{l-1}$ and $z_{l}, \theta(z)$ is the step function, and the angle brackets denote a time and ensemble average over the atom positions $r_{i}=\left(x_{i}, y_{i}, z_{i}\right)$. For the rest of the paper we shall restrict ourselves to consider the first allowed Bragg peak $\mathbf{k}=(2,0,0) 2 \pi / d$, where $d$ is the nearest-neighbor $\mathrm{Al}-\mathrm{Al}$ distance. We therefore omit the $\mathbf{k}$ index in the following. The structure factor in Eq. (1) can be used as a measure of the local order. In Fig. 1 it is clearly seen how the order decreases with increasing temperature, first slowly, because of incoherence induced by the lattice vibrations, and later suddenly, indicating the complete loss of order.
The sudden loss of order is not merely anomalous increase in the vibrational amplitude of an otherwise perfect lattice. This evidenced by the trajectories shown in Fig. 2. For the lower layers it is also evidenced by a jump in the total energy per atom of $0.1 \mathrm{eV}$ (to be compared with the bulk latent heat of melting of $0.11 \mathrm{eV} .^{15}$ We are thus lead to believe that we are actually seeing a layer-by-layer melting process.

At present we do not have enough data for an accurate determination of the melting temperature. It is, however, clear from Fig. 1 that the melting temperature of a layer increases with increasing distance from the surface. We have not reached the bulk value completely, and for the lowest layers the effect of the static substrate is important. The figure would indicate a value of $T_{M}$ just above $800 \mathrm{~K}$. This is not unreasonable compared with the experimental value of $933 \mathrm{~K},{ }^{15}$ given the approximations in the effective medium theory and in the implementation of the present calculations. We remark that although the static layers affect the lowest of the dynamical layers, they are sufficiently removed from the surface region of primary interest here.

Figure 1 indicates that the melting takes place in two stages. First, the first three layers melt at a temperature $\simeq 200 \mathrm{~K}$ below $T_{M}$, and then the rest comes gradually much closer to $T_{M}{ }^{16}$ This qualitatively explains the ion-scattering experiments of Frenken and van der Veen. ${ }^{1}$ Blackenhagen, Schommers, and Voegele ${ }^{2}$ have measured the temperature dependence of the (20) LEED intensity for $\mathrm{Al}(110)$, and find a rapid drop in intensity around $600 \mathrm{~K}$. In order to compare the calculated structure factors directly with the measured LEED intensities, they must be weighted by the probing depth of the scattering electrons. We do this by calculating a scattering intensity [again only the (20) peak will be discussed]

$$
I(T)=\left|\int S(z, T) P(z) \exp (-z / \lambda) d z\right|^{2} .
$$

Here $S(z, T)$ is defined as the structure factor for atoms in a small region around $z$ in analogy with the definition Eq. (1), $P(z)$ is the probability density of our finding an atom at $z$, and the exponential mimics the penetration depth of the LEED experiment. We use $\lambda=4.5 \AA$, which is typical for low-energy electrons. It must be pointed out that the calculated intensity of course does not include any of the multiple-scattering effects present in the real LEED experiment, but we do not expect this to affect the temperature dependence of the intensities.

The comparison of the calculated and experimental intensities is shown in Fig. 3. They are seen to be very similar except that the disorder starts about $100 \mathrm{~K}$ lower in the simulation than in the experiment. This is not surprising in view of the fact that, as found above, the potential used here gives a melting temperature that is somewhat too low.

The ion-scattering studies of $\mathrm{Pb}$ show a marked difference in the tendency towards premelting with crys- 

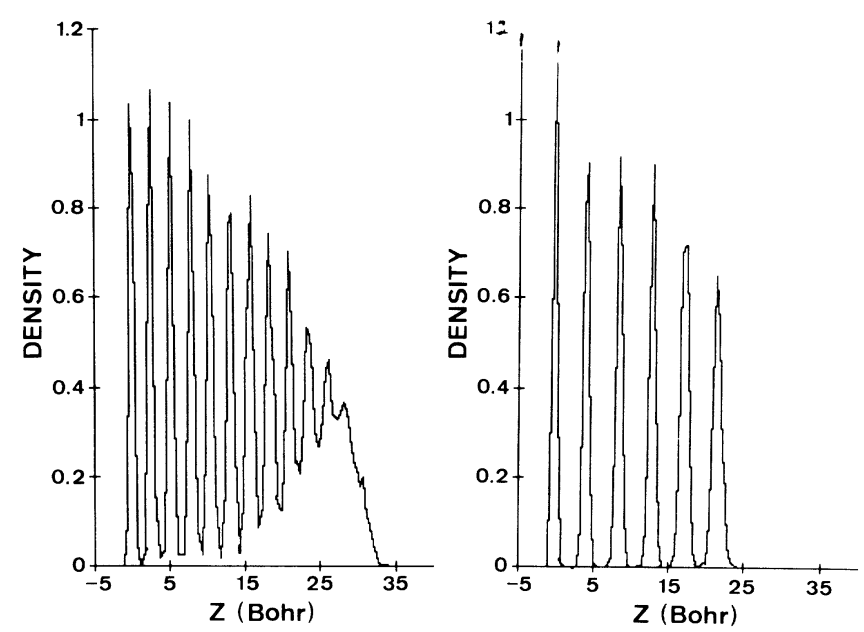

FIG. 4. Comparison of the atom density projected onto the $z$ axis for $\mathrm{Al}(110)$ and $(111)$ at $600 \mathrm{~K}$. The (110) surface is seen to show strong disordering of the first layers while the (111) surface has its layer structure preserved. The picture is qualitatively the same at $700 \mathrm{~K}$.

tal plane. The open (110) surface shows a strong effect starting about $150 \mathrm{~K}$ below $T_{M}$, as is also found above for $\mathrm{Al}(110)$, whereas the close-packed (111) surface only showed a weak disordering about $50 \mathrm{~K}$ below $T_{M}{ }^{3}$ Our preliminary studies of the $\mathrm{Al}(111)$ surface indeed show much weaker disordering than that found for the (110) surface as shown in Fig. 4.

Detailed study of the atomic trajectories shows that the disordering we observe in connection with the melting transition is triggered by the formation of vacancies. Over the time scale of the simulation we never observe diffusion except when vacancies are present. One role of the surface in connection with the melting process may be to provide low-energy vacancies. The energy cost of the formation of a Frenkel pair on the (110) surface is only $0.3 \mathrm{eV}$. On the (111) surface, on the other hand, where we see only weak signs of melting below the bulk melting point, the same energy is $1.3 \mathrm{eV}$. In the bulk the corresponding energy is much larger $(4.2 \mathrm{eV})$.

In conclusion, we have shown that the premelting of the open $\mathrm{Al}(110)$ surface can be adequately simulated by use of the effective-medium theory to calculate the total energy of the system. We see definite signs of a gradual layer-by-layer melting up to $200 \mathrm{~K}$ below the bulk melting point. The disordering we tentatively associate with the very small vacancy formation energy at this surface.

Financial support from European Economic Community twinning Grant No. ST2J-0286-C(TT) (P.S. and J.K.N.) U. S. Department of Energy Grant No. FG0587ER45234 (U.L.) is gratefully acknowledged.

${ }^{1}$ J. W. M. Frenken and J. F. van der Veen, Phys. Rev. Lett. 54, 134 (1985).

${ }^{2}$ P. von Blackenhagen, W. Schommers, and V. Voegele, J. Vac. Sci. Technol. A 5, 649 (1987).

${ }^{3}$ B. Pluis, A. W. Dernier van der Gon, J. W. M. Frenken, and J. F. van der Veen, Phys. Rev. Lett. 59, 2678 (1987).

${ }^{4}$ A. R. Ubbelohde, The Molten State of Matter (Wiley, New York, 1978), and references therein.

${ }^{5}$ C. S. Jayanthi, E. Tosatti, A. Fasolino, and L. Pietronero, Surf. Sci. 152/153, 155 (1985).

${ }^{6}$ R. Lipowsky and W. Speth, Phys. Rev. B 28, 3983 (1983).

${ }^{7}$ J. Q. Broughton and L. V. Woodcock, J. Chem. Phys. 79, 5119 (1983).

${ }^{8}$ R. M. Cotterill, Philos. Mag. 32, 1283 (1975).

${ }^{9}$ P. Carnevali, F. Ercolessi, and E. Tosatti, Phys. Rev. B 36, 6701 (1987).

${ }^{10}$ K. W. Jacobsen, J. K. Nørskov, and M. J. Puska, Phys. Rev. B 35, 7423 (1987).

${ }^{11}$ A. R. Williams and U. von Barth, in Theory of the Inhomogeneous Electron Gas, edited by S. Lundqvist and N. H. March (Plenum, New York, 1983).

${ }^{12}$ The parameter $\eta_{2}$ is, however, determined from the freeatom tail density as discussed in section VII.C of Ref. 10.

${ }^{13}$ P. Stoltze, K. W. Jacobsen, and J. K. Nørskov, Phys. Rev. B 36, 5035 (1987).

${ }^{14}$ Dieter W. Heermann, Computer Simulation Methods in Theoretical Physics (Springer-Verlag, Berlin, 1986).

${ }^{15}$ R. Hultgren, R. L. Orr, P. D. Anderson, and K. K. Kelly, Selected Values of Thermodynamic Properties of Metals and Alloys (Wiley, New York, 1963).

${ }^{16}$ A. Trayanov and E. Tosatti, Phys. Rev. Lett. 59, 2207 (1987). 